\title{
Liquid Biopsy for Colorectal Adenoma: Is the Exosomal miRNA Derived from Organoid a Potential Diagnostic Biomarker?
}

\section{Tomoyuki Handa}

Division of Gastroenterology, Tohoku University Graduate School of Medicine, Sendai, Japan Masatake Kuroha ( $\square$ kuroha@med.tohoku.ac.jp)

Division of Gastroenterology, Tohoku University Graduate School of Medicine, Sendai, Japan Hiroshi Nagai

Department of Gastroenterology, Shirakawa Kosei General Hospital, Fukushima, Japan

\section{Yusuke Shimoyama}

Division of Gastroenterology, Tohoku University Graduate School of Medicine, Sendai, Japan

\section{Takeo Naito}

Division of Gastroenterology, Tohoku University Graduate School of Medicine, Sendai, Japan

\section{Rintaro Moroi}

Division of Gastroenterology, Tohoku University Graduate School of Medicine, Sendai, Japan

\section{Yoshitake Kanazawa}

Division of Gastroenterology, Tohoku University Graduate School of Medicine, Sendai, Japan

Hisashi Shiga

Division of Gastroenterology, Tohoku University Graduate School of Medicine, Sendai, Japan

\section{Yoichi Kakuta}

Division of Gastroenterology, Tohoku University Graduate School of Medicine, Sendai, Japan

\section{Yoshitaka Kinouchi}

Health Administration Center, Center for the Advancement of Higher Education, Tohoku University, Sendai, Japan

\section{Atsushi Masamune}

Division of Gastroenterology, Tohoku University Graduate School of Medicine, Sendai, Japan

\section{Research}

Keywords: exosome, miR-1246, miR-1290, miR-4284, miR-4323

Posted Date: October 7th, 2020

DOI: https://doi.org/10.21203/rs.3.rs-84445/v1 
License: (c) (i) This work is licensed under a Creative Commons Attribution 4.0 International License. Read Full License

Version of Record: A version of this preprint was published at Clinical and Translational Gastroenterology on May 1st, 2021. See the published version at https://doi.org/10.14309/ctg.0000000000000356. 


\section{Abstract}

Background: MicroRNAs (miRNAs) have attracted considerable attention as tumor biomarkers. Nevertheless, few reports have described studies of colorectal adenoma (CRA) for liquid biopsy. Recently, organoid culture system development has supported the long-term expansion of investigations of human colon epithelium. This study examined the profile of exosomal miRNAs extracted from CRA organoid and potentiation of candidate miRNAs for clinical application of liquid biopsy in CRA patients.

Method: We established organoids from normal colon tissues and CRA from endoscopically resected specimens. Exosomes were prepared from the conditioned medium of the organoids. miRNAs were prepared from the exosomes and their source organoids. The microRNA expression profiles were compared using microarray analysis. To investigate exosomal miRNAs extracted from organoid culture as a candidate for liquid biopsy, we prospectively compared changes in the expression of serum and exosomal miRNA before and after endoscopic resection in 26 patients with CRA.

Result: We established three organoid lines from normal colon epithelium and CRA. Microarray analysis revealed that seven exosomal miRNAs expression in CRA organoids were increased compared with normal colon organoids: miR-4323, miR-4284, miR-1268a, miR-1290, miR-6766-3p, miR-21-5p, and miR1246. The expressions of four exosomal miRNAs (miR-4323, miR-4284, miR-1290, and miR-1246) and two serum miRNAs (miR-1290 and miR-1246) were significantly lower in post-treatment sera. Combinations of four exosomal miRNA expressions can differentiate both CRA and large-size $(>12.6$ $\left.\mathrm{cm}^{2}\right)$ CRA with respective areas under the curve (AUCs) of $0.698(95 \% \mathrm{Cl}=0.536-0.823)$ and $0.834(95 \%$ $\mathrm{Cl}=0.660-0.929)$. Combinations of two-serum miRNA expression can differentiate both CRA and largesize CRA with respective AUCs of $0.691(95 \% \mathrm{Cl}=0.528-0.817)$ and $0.834(95 \% \mathrm{Cl}=0.628-0.938)$.

Conclusion: This report is the first to show that exosomal miRNAs derived from CRA organoid culture have potential for use as diagnostic biomarkers for CRA.

\section{Introduction}

Colorectal cancer (CRC) is the third most common malignancy and the second leading cause of cancerrelated death worldwide[1]. CRC follows a linear progression from a normal colonic epithelium to adenoma initiation, carcinoma transformation, and metastasis [2]. Therefore, the detection and treatment of colorectal adenomas (CRA) can significantly reduce the risk of invasive colorectal tumors and mortality [3]. For early detection for CRC, stool-based screening with a fecal immunochemical test (FIT) is commonly used worldwide [4]. However, the tests have low sensitivity for adenomatous lesions (7.6$40 \%$ ) [5-7]. They are unsuitable for screening of CRA. In addition, colonoscopy is unsuitable as a screening because of its high invasiveness, cost, and low adherence [8]. It is necessary to establish a noninvasive and highly sensitive screening test of CRA for the prevention of CRC.

Recent studies have demonstrated that microRNAs (miRNAs) are secreted from various cells, including cancer cells, into bodily fluids such as blood, urine, breast milk, and saliva, either as free miRNAs or via 
exosomes [9]. miRNAs are noncoding single-stranded RNA molecules of 18 to 23 nucleotides in length, that promote mRNA cleavage and subsequent degradation by binding to the complementary $3^{\prime}$ untranslated region of the mRNA[10]. Actually, miRNAs have been implicated in the development and progression of CRC by functioning as oncogenes and tumor suppressors [11]. Exosomes are 50-100 nm nanoscale vesicles that contain diverse host cell-derived bioactive molecules such as proteins, lipids, and miRNAs [12]. There has been increasing interest in examining the contents of exosome-miRNAs as candidates for liquid biopsy biomarkers. Reportedly, serum exosome miRNAs such as let-7a, miR-1229, miR-1246, miR-150, miR-21, miR-223, miR-23a, and miR-486 are highly expressed in CRC patients and are regarded as having potential for use in liquid biopsy [9]. Nevertheless, few reports have described studies of CRA for liquid biopsy. One reason is that candidate miRNAs for liquid biopsy have been isolated from the culture supernatant of colon cancer cell lines originating from advanced colon cancer.

Development of three-dimensional organoid culture systems has supported long-term expansion of human normal colon epithelia, CRA, and CRC $[13,14]$. In organoid cultures, cancer stem cells are maintained in a microenvironment using a cocktail of niche factors supplemented in the medium, including Noggin, R-spondin, and epidermal growth factor. For this study, we investigated profiles of exosome miRNAs derived from CRA using long-term culture in three-dimensional organoids. Furthermore, we examined whether candidate miRNAs derived from CRA organoids have potential for use as diagnostic biomarkers for CRA.

\section{Materials And Methods}

\subsection{Patients}

This study examined patients who had been diagnosed as having CRA of more than $10 \mathrm{~mm}$ in diameter. Furthermore, all lesions were treated by endoscopic submucosal dissection (ESD). This study included data of 54 patients at Tohoku University Hospital between February 2018 and April 2019. Colorectal tumors were defined as adenoma in Categories 3 and 4 and as cancer in Category 5 according to the Vienna classification [15]. Patients were excluded from the study if they met one or more of the following criteria: past history of cancer in other organs, inflammatory disease such as connective tissue disease, and diagnosed as Category 5 by histological assessment after ESD. This study was approved by the Ethics committee of Tohoku University Hospital (nos. 2017-1-949, 2019-1-379). All patients provided written informed consent before participation.

\subsection{Research method}

This study was conducted in two phases. In the first phase study (Fig. 1A), we established organoids from the normal colon epithelium and CRA in pairs from endoscopically resected specimens at three patients. Then exosomes derived from each organoid were collected by ultracentrifugation. Then microarray analysis was conducted for each of the normal colon epithelium-derived organoids and CRAderived organoids. In the second phase study (Fig. 1B), we prospectively compared changes in the expression of serum and exosomal miRNA after endoscopic resection. Pre-treatment sera were obtained 
within one month prior to endoscopic resection. Post-treatment sera were obtained at six months after endoscopic resection. At the time, total colonoscopy was performed to confirm healing of the mucosa and lack of recurrence or residual in the treated area. The candidate miRNAs selected in the first phase were validated using real-time PCR (RT-PCR).

\subsection{Establishment of organoid cultures}

Organoid culture was performed based on protocols as previously reported [16], with slight modifications. In brief, tumor tissues were cut and carefully washed with ice-cold PBS. The tumor tissues were then incubated in a $37^{\circ} \mathrm{C}$ water bath for $30 \mathrm{~min}$ with $50 \mu \mathrm{g} / \mathrm{ml}$ Liberase (TH research grade; Roche Diagnostics Corp., Basel, Switzerland). The tumor cell pellets were then resuspended in Matrigel (BD Matrigel; Becton, Dickinson and Co., Franklin Lakes, NJ, USA) and were seeded onto 24-well plates. For normal colon tissues, the tissues were cut into small pieces; they were then washed carefully with ice-cold PBS. Normal tissue fragments were then incubated with $2.5 \mathrm{mM}$ EDTA chelation buffer for $1 \mathrm{hr}$ at $4{ }^{\circ} \mathrm{C}$. Next, by violently pipetting with PBS, crypts were released. The crypts were then resuspended in Matrigel and were seeded onto 24-well plates. Both the normal colon organoids and tumor organoids were cultured using organoid culture medium with niche factor advanced DMEM/F12 (Gibco, Grand Island, NY, USA) with penicillin-streptomycin (Gibco), 2 mM GlutaMAX (Gibco), 10 mM HEPES (Gibco), 2\% B-27 Supplement (Gibco), 1 mM N -acetylcysteine (Wako, Osaka, Japan), 50 ng/ml EGF (Gibco), 100 ng/ml Noggin (Peprotech, Rocky Hill, NJ), 10\% R-spondin-1 conditioned medium (Trevigen Inc., Gaithersburg), 10\% Afamin/Wnt3a (JSR Life Sciences, Tsukuba, Japan), 500 nm A83-01 (Wako), 10 nm Gastrin (SigmaAldrich, St. Louis, MO), and $10 \mu \mathrm{M}$ SB202190 (Cayman Chemical, Ann Arbor, MI)). Also, $10 \mu \mathrm{M}$ Y-27632 (Wako) was added to each well to the culture medium for establishment of organoid cultures after passage. The medium was replaced every 2-3 days, and organoids were passaged, generally on every 7 th day of culture.

\subsection{Exosome isolation from organoid-conditioned medium}

Exosomes were isolated from conditioned medium of organoids by ultracentrifugation [17]. After several passages, the organoid-conditioned medium was collected. The medium was first centrifuged at $300 \times g$ for $10 \mathrm{~min}$ and then at $2000 \times \mathrm{g}$ for $30 \mathrm{~min}$ to precipitate the cells. The supernatant was then filtered through $0.2 \mu \mathrm{m}$ filters and ultracentrifuged at $100000 \times g$ for $70 \mathrm{~min}$ to pellet exosomes, which were then washed in PBS. These vesicles were then ultracentrifuged at $100000 \times g$ for $70 \mathrm{~min}$. The final pellet was resuspended in $100 \mu \mathrm{PBS}$ and frozen at $-80^{\circ} \mathrm{C}$.

\subsection{Electron microscopic analysis}

Isolated exosomes were evaluated for their morphology and size distribution using transmission electron microscopy, as previously described [18]. Exosomes were fixed with $2 \%$ glutaraldehyde in $0.1 \mathrm{M}$ phosphate buffer, and were subsequently fixed in $2 \%$ osmium tetroxide for $2 \mathrm{hr}$. After washing with distilled water, dehydration through a graded series of ethanol, and embedding in epoxy resin at $60^{\circ} \mathrm{C}$, ultrathin sections were prepared using an ultramicrotome (LEICA EM UC7; Leica Microsystems Japan, 
Tokyo, Japan). They were then stained with uranyl acetate and lead citrate and examined using transmission electron microscopy (TEM, Hitachi H-7600; Hitachi High-Technologies, Tokyo, Japan).

\subsection{Nanoparticle tracking analysis}

The amount and size distribution of exosomes were analyzed using microscopy (Nanosight NS300; Malvern Panalytical, Malvern, United Kingdom). Each sample was diluted appropriately with PBS to give counts in the linear range of the instrument. The particles in the laser beam exhibit Brownian motion; video was recorded in duplicate for $60 \mathrm{~s}$. Data were then analyzed using NTA software (ver. 3.3; Malvern Panalytical, Malvern, United Kingdom).

\subsection{RNA isolation and microarray analysis}

Total RNAs including miRNAs were extracted using a kit (miRNeasy Mini Kit; Qiagen Inc., Hilden, Germany) according to the manufacturer's instructions. The RNA quality was assessed by microfluidic chip (Bioanalyzer 2100 Expert; Agilent Technologies, Santa Clara, CA) using a kit (RNA 6000 pico kit; Agilent Technologies). Microarray analysis was done (Agilent SurePrint G3 Human miRNA microarray kit $8 \times 60 \mathrm{~K}$, ver. Human_miRNA_V21,0; Agilent Technologies), 2 ng total RNAs from the exosome fractions were labeled using kits with a miRNA labeling reagent (Low Input Quick Amp Labeling Kit, RNA spike kit; Agilent Technologies). Then they were hybridized according to the manufacturer's instructions. After hybridization, the array was washed and scanned using a DNA microarray scanner (Agilent Technologies). After numerical conversion of the raw data using feature extraction software (ver. 10.7; Agilent Technologies), the transformed data were analyzed using software (GeneSpring ver. 12.5; Digital Biology).

\subsection{MiRNA and exosomal miRNA extraction from patients' serum}

From each participant, whole blood was taken and processed using centrifugation at $1900 \times g$ for $10 \mathrm{~min}$ centrifuge. To remove cell debris, the supernatant was centrifuged at $4{ }^{\circ} \mathrm{C}$ and $16,000 \times g$ for $10 \mathrm{~min}$ and then stored at $-80^{\circ} \mathrm{C}$. Total miRNA was extracted from serum samples using a kit (miRNeasy Serum/Plasma; Qiagen Inc.) according to the manufacturer's instructions. Exosomes was extracted from serum samples by using the polymer precipitation method (ExoQuick; System Biosciences, Palo Alto). Then, exosomal RNA was extracted using a kit (miRNAeasy Mini: Qiagen Inc.).

\section{9. qRT-PCR analysis of miRNAs}

Serum concentrations of six candidate miRNAs were quantified by reverse transcription polymerase chain reaction (RT-PCR) using a kit (Taqman MicroRNA Reverse Transcription; Applied Biosystems, Foster City, CA) and Taqman ${ }^{\text {TM }}$ miRNA Assay (Applied Biosystems). The RT-PCR was performed in triplicate using Lightcycler 96 system (Roche, Basel, Switzerland). The miR-16 was used as an internal control because it has been reported as a reliable endogenous control for RT-PCR analysis of miRNA in humans [19]. miRNA expression levels were normalized by miR-16 using the $2-\Delta C t$ method. 


\subsection{Statistical analyses}

Statistical analyses were performed using JMP ver. 14.0 (SAS Institute Inc., Tokyo, Japan). To compare miRNA expression in paired samples, $t$-tests were used. The values are presented as mean \pm standard deviation (SD). Significance was inferred for results found to have two-sided $P<0.05$. In graphs showing the relative expression of each miRNAs in the patient sera, diagnostic performance for each biomarker individually and in combination was evaluated using receiver operating characteristic (ROC) curve analysis and by reporting the area under the curve (AUC).

\section{Results}

\subsection{Isolation of exosomes from patient-derived organoids}

We established three organoid lines from normal colon epithelium and CRA in pairs from the specimen resected by endoscopy. Clinical characteristics of three patients are presented in Table 1. Paraffin sections of the organoids showed that the established organoids imitated the original structures as a budding form, as elucidated using $\mathrm{H}$-E staining (Fig. 2A). Exosomes were isolated from conditioned medium of the organoids by ultracentrifugation. Under transmission electron microscopy, the size of the vesicles was approximately $100 \mathrm{~nm}$ (Fig. 2B), which corresponded to the results of earlier studies [12]. The nanoparticle tracking analyzer showed that the mode size of the vesicles in isolated exosomes was $107.4 \pm 11.7$ nm (Fig. 2C).

Table 1

Characteristics of the patients from whom were established organoids

\begin{tabular}{|lllll|}
\hline & Age & Gender & Location of the tumor & Vienna classification \\
\hline 1 & 78 & Female & Left colon & Category 4 \\
\hline 2 & 81 & Male & Left colon & Category 4 \\
3 & 63 & Female & Right colon & Category 3 \\
\hline
\end{tabular}

\subsection{Microarray analysis revealed profiles of exosomal miRNA derived from CRA organoids}

Next, we isolated exosomal miRNAs derived from normal colon epithelium organoids and CRA organoids. We confirmed that the isolated exosomal miRNAs did not include ribosomal RNA using a Bioanalyzer (Fig. 3A). In exosomal miRNA, the expressions of 15 miRNAs were found to be higher, whereas 97 miRNAs were found to be lower in the CRA-derived organoids than those of normal epithelium-derived organoids (Fig. 3B). For CRA-derived organoids, 10 miRNA levels (miR-4323, miR-4284, miR-1268a, miR1290, miR-6766-3p, miR-21-5p, miR-1246, miR-2278, miR-3148, mir-595) were found to be higher, with FC $>0.5$; also, 75 miRNA levels were lower, with FC>0.5. Among these miRNAs with decreased levels, 12 
miRNAs were found to be lower, with FC > 3: miR-633, miR-6087, miR-4443, miR-5703, miR-6833-5p, miR711, miR-7150, miR-6133, miR-4534, miR-6865-5p, miR-6775-5p, and miR-4716-3p (Table 2). From 10 miRNAs showing increased levels in the CRA-derived organoid, we selected 7 candidate miRNAs for the second phase study: 5 miRNAs (miR-4323, miR-4284, miR-1268a, miR-1290 and miR-6766-3p) that were found to have higher levels with FC $>1$ and two miRNAs (miR-21-5p, miR-1246) that were found in an earlier study to have association with colon cancer $[9,20]$.

Table 2

miRNAs for which expression was changed in exosomes between adenoma and normal epithelium by microarray analysis

\begin{tabular}{|c|c|c|c|c|c|}
\hline \multicolumn{6}{|l|}{ Exosomal miRNA } \\
\hline \multicolumn{3}{|l|}{ Adenoma/Normal } & \multicolumn{3}{|l|}{ Normal/Adenoma } \\
\hline Systematic name & $\log _{2} \mathrm{FC}$ & $p$ value & Systematic name & $\log _{2} \mathrm{FC}$ & $p$ value \\
\hline hsa-miR-4323 & 2.21 & 0.18 & hsa-miR-630 & 4.71 & $<0.01$ \\
\hline hsa-miR-4284 & 2.21 & 0.18 & hsa-miR-6087 & 4.61 & $<0.01$ \\
\hline hsa-miR-1268a & 2.06 & 0.25 & hsa-miR-4442 & 4.23 & 0.01 \\
\hline hsa-miR-1290 & 1.41 & 0.03 & hsa-miR-5703 & 4.11 & 0.08 \\
\hline hsa-miR-6766-3p & 1.29 & 0.53 & hsa-miR-6833-5p & 3.47 & 0.07 \\
\hline hsa-miR-21-5p & 0.89 & 0.70 & hsa-miR-711 & 3.42 & $<0.01$ \\
\hline hsa-miR-1246 & 0.86 & 0.09 & hsa-miR-7150 & 3.37 & 0.08 \\
\hline hsa-miR-2278 & 0.74 & 0.63 & hsa-miR-6133 & 3.24 & 0.08 \\
\hline hsa-miR-3148 & 0.70 & 0.75 & hsa-miR-4534 & 3.15 & 0.18 \\
\hline \multirow[t]{3}{*}{ hsa-miR-595 } & 0.52 & 0.76 & hsa-miR-6865-5p & 3.08 & 0.09 \\
\hline & & & hsa-miR-6775-5p & 3.07 & 0.10 \\
\hline & & & hsa-miR-4716-3p & 3.06 & 0.10 \\
\hline FC, fold change & & & & & \\
\hline
\end{tabular}

\subsection{Four exosomal miRNAs and two serum miRNAs lower after endoscopic resection}

To clarify the diagnostic value for CRA, we examined the expression of candidate miRNAs from CRA patients. Initially, 54 patients were enrolled in this study. Then 13 patients were excluded because they had a history of cancer or inflammatory disease. Another six patients were excluded because they were diagnosed as having a tumor of Category 5 by histopathological assessment of resected specimens. Six 
patients were excluded because serum after treatment was not available. Finally, 26 patients were analyzed in this study (Fig. 4A). Clinical characteristics of 26 patients are presented in Table 3. Because primers of mir-1268a could not be created with sufficient accuracy (data not shown), we validated the expression changes between pre-treatment sera and post-treatment sera of six miRNAs using RT-PCR: miR-4323, miR-4284, miR-1290, miR-6766-3p, miR-21, and miR-1246. In exosomal miRNAs, four miRNAs were found to be lower levels in post-treatment serum than in pre-treatment serum: miR-1290, miR-1246, miR-4323, and miR-4284 (Fig. 4B). In serum miRNAs, two miRNAs (miR-1290 and miR-1246) were found to have lower levels in post-treatment serum than in pre-treatment serum (Fig. 4C). 
Table 3

Clinical characteristics of the patients from whom serum was collected before and after endoscopic treatment

\section{Characteristics}

Patients $(n=26)$

Age, $n(\%)$

$\begin{array}{ll}<70 \text { years } & 13(50.0) \\ \geq 70 \text { years } & 13(50.0)\end{array}$

Gender, $n(\%)$

Male

Female

Location, $n(\%)$

$\begin{array}{ll}\text { Right colon } & 19(73.1) \\ \text { Left colon } & 7(26.9)\end{array}$

Macroscopic type, $n(\%)$

$\begin{array}{ll}\text { Protruding } & 3(11.5) \\ \text { LST-G } & 15(57.7) \\ \text { LST-NG } & 8(30.8)\end{array}$

Tumor size, $n(\%)$ *

$\begin{array}{ll}<12.6 \mathrm{~cm}^{2} & 13(50.0) \\ \geq 12.6 \mathrm{~cm}^{2} & 13(50.0)\end{array}$

Vienna classification, $n(\%)$

$\begin{array}{ll}\text { Category } 3 & 14(53.8) \\ \text { Category } 4 & 12(46.2)\end{array}$

LST-G, laterally spreading tumor granular type; LST-NG, laterally spreading tumor non-granular type. * Tumor size was assessed by elliptical area and was calculated from long and short axes (cm) in resected specimens.

Next, we examined whether high expression of miRNAs is associated with clinical characteristics. The high expression of serum mir1290 was found from univariate and multivariate analyses to be associated with adenoma size. No other miRNA (either exosomal or serosal) was found to be associated with clinical characteristics (Table 4). 
Table 4

Clinical factors with high expression of serum miR-1290

High expression of serum miR-1290

$(n=13)$
Univariate $^{*}$ Multivariate $^{\star *}$

P-value
$P$ value

Age, $n(\%)$

$<70$ years

$6 / 13(46.1)$

1.00

$\geq 70$ years

$7 / 13(53.8)$

Gender, $n(\%)$

Male

$5 / 10(50.0)$

1.00

Female

$8 / 16(50.0)$

Location, $n(\%)$

Right colon

7/19 (36.8)

0.07

Reference

Left colon

6/7 (85.7)

$5.59(0.26-$ 118.23)

Macroscopic type, $n$

(\%)

Protruding

1/3 (33.3)

0.14

Reference

LST-G

$10 / 15(66.7)$

$15.6(0.34-$

710.67)

LST-NG

$2 / 8(25.0)$

$11.3(0.15-$ 829.61)

Tumor size, $n(\%)$

$<12.6 \mathrm{~cm}^{2}$

11/13 (84.6)

$<0.01$

Reference

$\geq 12.6 \mathrm{~cm}^{2}$

2/13 (15.4)

33.1 (1.97556.64)

0.16

0.27

Vienna classification, $n(\%)$

Category 3

$6 / 14(42.9)$

0.70

Factors with $P$ value of less than 0.2 in univariate analysis were included in multivariate analyses: LST-G, laterally spreading tumor granular type; LST-NG, laterally spreading tumor non-granular type; $\mathrm{OR}$, odds ratio; $\mathrm{Cl}$, confidence interval. ${ }^{*}$ Chi-square test or Fisher's exact probability test. **multiple logistic regression analysis 


\section{High expression of serum miR-1290 \\ $(n=13)$}

Univariate $^{*}$ Multivariate ${ }^{* *}$

Pvalue

Category 4

$7 / 12(58.3)$

Factors with $P$ value of less than 0.2 in univariate analysis were included in multivariate analyses: LST-G, laterally spreading tumor granular type; LST-NG, laterally spreading tumor non-granular type; $\mathrm{OR}$, odds ratio; $\mathrm{Cl}$, confidence interval. ${ }^{*}$ Chi-square test or Fisher's exact probability test. **multiple logistic regression analysis

\subsection{Diagnostic ability of candidate miRNAs as biomarkers for CRA}

We generated ROC curves to assess the usefulness of selected miRNAs as potential biomarkers for CRA (Fig. 5). Regarding exosomal miRNAs, ROC analyses revealed miR-4323, miR-4284, miR1290, and miR1246 levels as significantly discriminating factors indicating the presence of CRA in patient with respective AUC values of 0.637 (95\% confidence interval $(\mathrm{Cl})=0.470-0.776), 0.677(95 \% \mathrm{Cl}=0.514-$ $0.805), 0.694(95 \% \mathrm{Cl}=0.534-0.818)$ and $0.635(95 \% \mathrm{Cl}=0.472-0.772)$. The AUC for combined values of four exosomal miRNAs was $0.698(95 \% \mathrm{Cl}=0.536-0.823)$. Next, we attempted to identify patients with large adenoma (approx. $\geq 12.6 \mathrm{~cm}^{2}$; median tumor size), for whom ROC analyses revealed combined values of four exosomal miRNAs with AUC values as $0.834(95 \% \mathrm{Cl}=0.660-0.929)$.

Regarding serum miRNA, ROC analyses used miR-1290 and miR-1246 levels as significant factors for discriminating the presence of CRA with respective AUC values of $0.705(95 \% \mathrm{Cl}=0.544-0.827), 0.639$ $(95 \% \mathrm{Cl}=0.476-0.776)$. The AUC for combined values of two miRNAs was $0.691(95 \% \mathrm{Cl}=0.528-0.817)$. In patients with large adenomas, ROC analyses revealed combined values of two serum miRNAs with AUC values of $0.834(95 \% \mathrm{Cl}=0.628-0.938)$.

\section{Discussion}

In this study, we extracted exosomal miRNA from three-dimensional organoid culture and elucidated profiles of exosomal miRNAs from CRA organoids. Reportedly, the expression of exosomal miRNAs such as miR-21, miR-1246, miR-1290, miR-23a, miR-200c, miR-203a, miR-17, miR-19a and miR-7641 are increased in CRC [21-26]. Although expressions of miR-21, miR-1246, and miR-1290 are reportedly increased in CRC and CRA, no report has described the increased expression of miR-4323, miR-4284, miR1268a, or miR-6766-3p in CRA. These miRNAs might be related to mutation of the APC gene, which causes adenoma initiation in the colonic epithelium[27]. Results also suggest that expression of exosomal miRNAs is altered along with progress of stages of colorectal tumors.

To our knowledge, no study has examined exosomal miRNAs extracted from organoid culture as a candidate for liquid biopsy. The AUCs in CRA patients were 0.698 for the combination of exosomal 
miRNAs and 0.698 for the combination of serum miRNAs. Moreover, the AUCs in patients with larger CRA (approx. $\geq 12.6 \mathrm{~cm}^{2}$ ) were 0.834 for the combination of exosomal miRNAs and 0.834 for the combination of serum miRNAs. The AUC in FIT for advanced adenoma (those $\geq 10 \mathrm{~mm}$ size or with villous and/or high-grade dysplasia) is reportedly about 0.62 [6]. The diagnostic capacity of liquid biopsy using miRNAs presented in this report is presumed to be higher. Furthermore, the results, demonstrating that better diagnostic capacity was obtained for larger tumors, suggests that the miRNAs used as candidates are secreted by tumors. Since it has been reported that the larger the size of adenoma, the more often complicated with cancer[28], it is expected that the candidate miRNAs especially useful as a screening for adenomas at high risk of cancer. However, the accuracy of liquid biopsy must be improved, particularly in consideration of the labor and costs of miRNA extraction and PCR testing. In this study, exosomes were extracted from the organoid culture supernatant by ultracentrifugation because few exosomes contained in the culture supernatant and required a large amount of medium. On the other hand, in a prospective study using patient sera, the polymer precipitation method was used. The available exosome kinds differ depending on the separation method [29, 30]. Presumably, miRNAs can be more effective biomarkers for CRA if it is possible to separate and extract from more tumor-derived exosomes. Result of our study showed no difference in miR-4283 or miR-4323 in serum miRNA levels: differences in expression were observed only for exosomal miRNAs. One influential factor might be that the change in expression was detected more sensitively because exosomes were extracted selectively.

In this prospective study, the expression of miRNAs in sera or serum exosomes of CRA patients were compared before and after endoscopic resection. Although differences in serum miRNAs before and after surgical resection in advanced CRC have been reported [9,31], no report of the literature has described comparison of serum miRNA levels before and after endoscopic resection of adenoma. Because factors other than the tumor can be excluded, that method can be regarded as yielding more accurate study results. The expressions of serum exosomal miR-21, miR-29a, miR-92a have been reported for use of biomarkers for CRA patients [32]. In this study, no difference was observed for miR-21 in the serum before and after treatment. Moreover, secretion of miR-29a or miR-92a from adenomatous organoids was not observed, possibly because of different extraction methods. Additionally, miR-29a and miR-92a might be secreted by cells other than tumor cells, such as fibroblasts and immune cells.

Reportedly, miR-4323 has been expressed less in patients of rectal cancer than in the normal colorectal epithelium [33], and it has also been expressed less in sera of patients with CRC than in healthy persons [34]. One report describes that miR-4284 was enriched in the cyst fluid from pancreatic intraductal papillary mucinous neoplasm with low grade dysplasia, but it was depleted in the cyst fluids derived from invasive pancreatic carcinomas. [35]. The expression of miR-4323 and miR-4284 might be lower in highly malignant tumor. Some reports have described that miR-1290 is expressed at a higher level in patients with CRC [36], and that it is related to epithelial mesenchymal transition [37], with miR-1246 promotes development of CRC via the expression of TGF- $\beta$ [38] and as a diagnostically useful biomarker of CRC and recurrence $[9,20,39,40]$. 
This study has several limitations. First, the investigation was conducted in a small number of patients and single center study. Second, studies of tumor lesions other than colorectal tumors are also need to clearly that candidate miRNAs are specific for patients with CRA. Finally, although serum at 6 months after endoscopic treatment was used as the control, the duration until the serum miRNA level returns to normal was not fully investigated.

\section{Conclusions}

In summary, this study elucidated a profile of exosomal miRNAs in CRA obtained using organoid culture. Results show that the extracted candidate miRNAs might be applicable to liquid biopsy. Organoid culture has been applied to not only the colorectal tumors but also tumors of various organs [41-43]. Using this technology, exosomal miRNAs could be expected from tumors of various organs at different stages and to create candidate miRNAs for liquid biopsy.

\section{Abbreviations}

CRC, colorectal cancer; FIT, fecal immunochemical test; CRA, colorectal adenoma; miRNAs, microRNAs; $E S D$, endoscopic submucosal dissection; qRT-PCR, quantitative reverse transcription-polymerase chain reaction; SD, standard error; ROC, receiver operating characteristic; $A U C$, area under the curve; $\mathrm{H}-\mathrm{E}$, hematoxylin-eosin; $\mathrm{FC}$, fold change; $\mathrm{Cl}$, confidence interval

\section{Declarations}

\section{Ethics approval and consent to participate}

This study was approved by the Ethics Committee of Tohoku University Graduate School of Medicine ((nos. 2017-1-949, 2019-1-379). All patients provided written informed consent.

\section{Consent for publication}

Not applicable

\section{Availability of data and material}

The datasets during and/or analyzed during the current study available from the corresponding author on reasonable request

\section{Competing interests}

The authors have no conflict of interest related to this report or the study it describes.

\section{Funding source}

This study was supported by JSPS KAKENHI Grant number JP19K17421. 
Author contributions

Tomoyuki Handa and Masatake Kuroha designed the study and wrote the initial draft of the manuscript. Yoichi Kakuta and Atsushi Masamune contributed to the analysis and interpretation of data and assisted in the preparation of the manuscript. All other authors have contributed to data collection and interpretation and critically reviewed the manuscript. All authors approved the final version of the manuscript and agreed to be accountable for all aspects of the work in ensuring that questions related to the accuracy or investigated and resolved.

\section{Acknowledgments}

We acknowledge technical support received from the Biomedical Research Core of Tohoku University Graduate School of Medicine.

\section{References}

1. Keum N, Giovannucci E: Global burden of colorectal cancer: emerging trends, risk factors and prevention strategies. Nat Rev Gastroenterol Hepatol 2019, 16:713-732.

2. Vogelstein B, Papadopoulos N, Velculescu VE, Zhou S, Diaz LA, Jr., Kinzler KW: Cancer genome landscapes. Science 2013, 339:1546-1558.

3. Zauber AG, Winawer SJ, O'Brien MJ, Lansdorp-Vogelaar I, van Ballegooijen M, Hankey BF, Shi W, Bond JH, Schapiro M, Panish JF, et al: Colonoscopic polypectomy and long-term prevention of colorectal-cancer deaths. N Engl J Med 2012, 366:687-696.

4. Navarro M, Nicolas A, Ferrandez A, Lanas A: Colorectal cancer population screening programs worldwide in 2016: An update. World J Gastroenterol 2017, 23:3632-3642.

5. Berger BM, Levin B, Hilsden RJ: Multitarget stool DNA for colorectal cancer screening: a review and commentary on the united states preventive services draft guidelines. World J Gastrointest Oncol 2016, 8:450-458.

6. Imperiale TF, Gruber RN, Stump TE, Emmett TW, Monahan PO: Performance characteristics of fecal Immunochemical tests for colorectal cancer and advanced adenomatous polyps: a systematic review and meta-analysis. Ann Intern Med 2019, 170:319-329.

7. Imperiale TF, Ransohoff DF, Itzkowitz SH, Levin TR, Lavin P, Lidgard GP, Ahlquist DA, Berger BM: Multitarget stool DNA testing for colorectal-cancer screening. N Eng/ J Med 2014, 370:1287-1297.

8. Inadomi JM, Vijan S, Janz NK, Fagerlin A, Thomas JP, Lin YV, Muñoz R, Lau C, Somsouk M, El-Nachef $\mathrm{N}$, Hayward RA: Adherence to colorectal cancer screening: a randomized clinical trial of competing strategies. Arch Intern Med 2012, 172:575-582.

9. Ogata-Kawata H, Izumiya M, Kurioka D, Honma Y, Yamada Y, Furuta K, Gunji T, Ohta H, Okamoto H, Sonoda H, et al: Circulating exosomal microRNAs as biomarkers of colon cancer. PLoS One 2014, 9:e92921.

10. Bartel DP: MicroRNAs: target recognition and regulatory functions. Cel/2009, 136:215-233. 
11. Yuan Q, Cao G, Li J, Zhang Y, Yang W: MicroRNA-136 inhibits colon cancer cell proliferation and invasion through targeting liver receptor homolog-1/Wnt signaling. Gene 2017, 628:48-55.

12. Choi DS, Kim DK, Kim YK, Gho YS: Proteomics, transcriptomics and lipidomics of exosomes and ectosomes. Proteomics 2013, 13:1554-1571.

13. Fujii M, Shimokawa M, Date S, Takano A, Matano M, Nanki K, Ohta Y, Toshimitsu K, Nakazato Y, Kawasaki K, et al: A colorectal tumor organoid library demonstrates progressive loss of niche factor requirements during tumorigenesis. Cell Stem Cell 2016, 18:827-838.

14. Sato T, Stange DE, Ferrante M, Vries RG, Van Es JH, Van den Brink S, Van Houdt WJ, Pronk A, Van Gorp J, Siersema PD, Clevers H: Long-term expansion of epithelial organoids from human colon, adenoma, adenocarcinoma, and Barrett's epithelium. Gastroenterology 2011, 141:1762-1772.

15. Schlemper RJ, Riddell RH, Kato Y, Borchard F, Cooper HS, Dawsey SM, Dixon MF, Fenoglio-Preiser CM, Flejou JF, Geboes K, et al: The Vienna classification of gastrointestinal epithelial neoplasia. Gut 2000, 47:251-255.

16. Fujii M, Matano M, Nanki K, Sato T: Efficient genetic engineering of human intestinal organoids using electroporation. Nat Protoc 2015, 10:1474-1485.

17. Gatti S, Bruno S, Deregibus MC, Sordi A, Cantaluppi V, Tetta C, Camussi G: Microvesicles derived from human adult mesenchymal stem cells protect against ischaemia-reperfusion-induced acute and chronic kidney injury. Nephrol Dial Transplant 2011, 26:1474-1483.

18. Masamune A, Yoshida N, Hamada S, Takikawa T, Nabeshima T, Shimosegawa T: Exosomes derived from pancreatic cancer cells induce activation and profibrogenic activities in pancreatic stellate cells. Biochem Biophys Res Commun 2018, 495:71-77.

19. Huang Z, Huang D, Ni S, Peng Z, Sheng W, Du X: Plasma microRNAs are promising novel biomarkers for early detection of colorectal cancer. Int J Cancer 2010, 127:118-126.

20. Jin G, Liu Y, Zhang J, Bian Z, Yao S, Fei B, Zhou L, Yin Y, Huang Z: A panel of serum exosomal microRNAs as predictive markers for chemoresistance in advanced colorectal cancer. Cancer Chemother Pharmacol 2019, 84:315-325.

21. Chiba M, Kimura M, Asari S: Exosomes secreted from human colorectal cancer cell lines contain mRNAs, microRNAs and natural antisense RNAs, that can transfer into the human hepatoma HepG2 and lung cancer A549 cell lines. Oncol Rep 2012, 28:1551-1558.

22. Clancy C, Khan S, Glynn CL, Holian E, Dockery P, Lalor P, Brown JA, Joyce MR, Kerin MJ, Dwyer RM: Screening of exosomal microRNAs from colorectal cancer cells. Cancer Biomark 2016, 17:427-435.

23. Ji H, Chen M, Greening DW, He W, Rai A, Zhang W, Simpson RJ: Deep sequencing of RNA from three different extracellular vesicle (EV) subtypes released from the human LIM1863 colon cancer cell line uncovers distinct miRNA-enrichment signatures. PLoS One 2014, 9:e110314.

24. Moshammer MI, Kalipciyan M, Bartsch R, Steger GG, Sedivy R, Mader RM: Exosomal microRNA transfer varies with specific microRNAs functional in colorectal cancer and cellular differentiation. Int J Clin Pharmacol Ther 2014, 52:87-88. 
25. Fu F, Jiang W, Zhou L, Chen Z: Circulating Exosomal miR-17-5p and miR-92a-3p Predict Pathologic Stage and Grade of Colorectal Cancer. Transl Oncol 2018, 11:221-232.

26. Szvicsek Z, Oszvald Á, Szabó L, Sándor GO, Kelemen A, Soós A, Pálóczi K, Harsányi L, Tölgyes T, Dede $\mathrm{K}$, et al: Extracellular vesicle release from intestinal organoids is modulated by Apc mutation and other colorectal cancer progression factors. Cell Mol Life Sci 2019, 76:2463-2476.

27. Nagel R, le Sage C, Diosdado B, van der Waal M, Oude Vrielink JA, Bolijn A, Meijer GA, Agami R: Regulation of the adenomatous polyposis coli gene by the miR-135 family in colorectal cancer. Cancer Res 2008, 68:5795-5802.

28. Winawer SJ, Zauber AG, Ho MN, O'Brien MJ, Gottlieb LS, Sternberg SS, Waye JD, Schapiro M, Bond $\mathrm{JH}$, Panish JF, et al.: Prevention of colorectal cancer by colonoscopic polypectomy. the national polyp study workgroup. N Engl J Med 1993, 329:1977-1981.

29. Buschmann D, Kirchner B, Hermann S, Märte M, Wurmser C, Brandes F, Kotschote S, Bonin M, Steinlein OK, Pfaffl MW, et al: Evaluation of serum extracellular vesicle isolation methods for profiling miRNAs by next-generation sequencing. J Extracell Vesicles 2018, 7:1481321.

30. Coumans FAW, Brisson AR, Buzas El, Dignat-George F, Drees EEE, El-Andaloussi S, Emanueli C, Gasecka A, Hendrix A, Hill AF, et al: Methodological Guidelines to Study Extracellular Vesicles. Circ Res 2017, 120:1632-1648.

31. Toiyama Y, Takahashi M, Hur K, Nagasaka T, Tanaka K, Inoue Y, Kusunoki M, Boland CR, Goel A: Serum miR-21 as a diagnostic and prognostic biomarker in colorectal cancer. J Natl Cancer Inst 2013, 105:849-859.

32. Uratani R, Toiyama Y, Kitajima T, Kawamura M, Hiro J, Kobayashi M, Tanaka K, Inoue Y, Mohri Y, Mori $T$, et al: Diagnostic potential of cell-free and exosomal microRNAs in the identification of patients with high-risk colorectal adenomas. PLoS One 2016, 11:e0160722.

33. Pellatt DF, Stevens JR, Wolff RK, Mullany LE, Herrick JS, Samowitz W, Slattery ML: Expression profiles of miRNA subsets distinguish human colorectal carcinoma and normal colonic mucosa. Clin Trans/ Gastroenterol 2016, 7:e152.

34. Zhang Y, Li M, Ding Y, Fan Z, Zhang J, Zhang H, Jiang B, Zhu Y: Serum MicroRNA profile in patients with colon adenomas or cancer. BMC Med Genomics 2017, 10:23.

35. Wang J, Paris PL, Chen J, Ngo V, Yao H, Frazier ML, Killary AM, Liu CG, Liang H, Mathy C, et al: Next generation sequencing of pancreatic cyst fluid microRNAs from low grade-benign and high gradeinvasive lesions. Cancer Lett 2015, 356:404-409.

36. Wu J, Ji X, Zhu L, Jiang Q, Wen Z, Xu S, Shao W, Cai J, Du Q, Zhu Y, Mao J: Up-regulation of microRNA-1290 impairs cytokinesis and affects the reprogramming of colon cancer cells. Cancer Lett 2013, 329:155-163.

37. Kim G, An HJ, Lee MJ, Song JY, Jeong JY, Lee JH, Jeong HC: Hsa-miR-1246 and hsa-miR-1290 are associated with stemness and invasiveness of non-small cell lung cancer. Lung Cancer 2016, 91:1522. 
38. Cooks T, Pateras IS, Jenkins LM, Patel KM, Robles Al, Morris J, Forshew T, Appella E, Gorgoulis VG, Harris CC: Mutant p53 cancers reprogram macrophages to tumor supporting macrophages via exosomal miR-1246. Nat Commun 2018, 9:771.

39. Imaoka H, Toiyama Y, Fujikawa H, Hiro J, Saigusa S, Tanaka K, Inoue Y, Mohri Y, Mori T, Kato T, et al: Circulating microRNA-1290 as a novel diagnostic and prognostic biomarker in human colorectal cancer. Ann Oncol 2016, 27:1879-1886.

40. Liu X, Xu X, Pan B, He B, Chen X, Zeng K, Xu M, Pan Y, Sun H, Xu T, et al: Circulating miR-1290 and miR-320d as Novel Diagnostic Biomarkers of Human Colorectal Cancer. J Cancer 2019, 10:43-50.

41. Dedhia PH, Bertaux-Skeirik N, Zavros Y, Spence JR: Organoid models of human gastrointestinal development and disease. Gastroenterology 2016, 150:1098-1112.

42. Lancaster MA, Renner M, Martin CA, Wenzel D, Bicknell LS, Hurles ME, Homfray T, Penninger JM, Jackson AP, Knoblich JA: Cerebral organoids model human brain development and microcephaly. Nature 2013, 501:373-379.

43. Takasato M, Er PX, Becroft M, Vanslambrouck JM, Stanley EG, Elefanty AG, Little MH: Directing human embryonic stem cell differentiation towards a renal lineage generates a self-organizing kidney. Nat Cell Biol 2014, 16:118-126.

\section{Figures}


Figure 1

(A)

same specimen

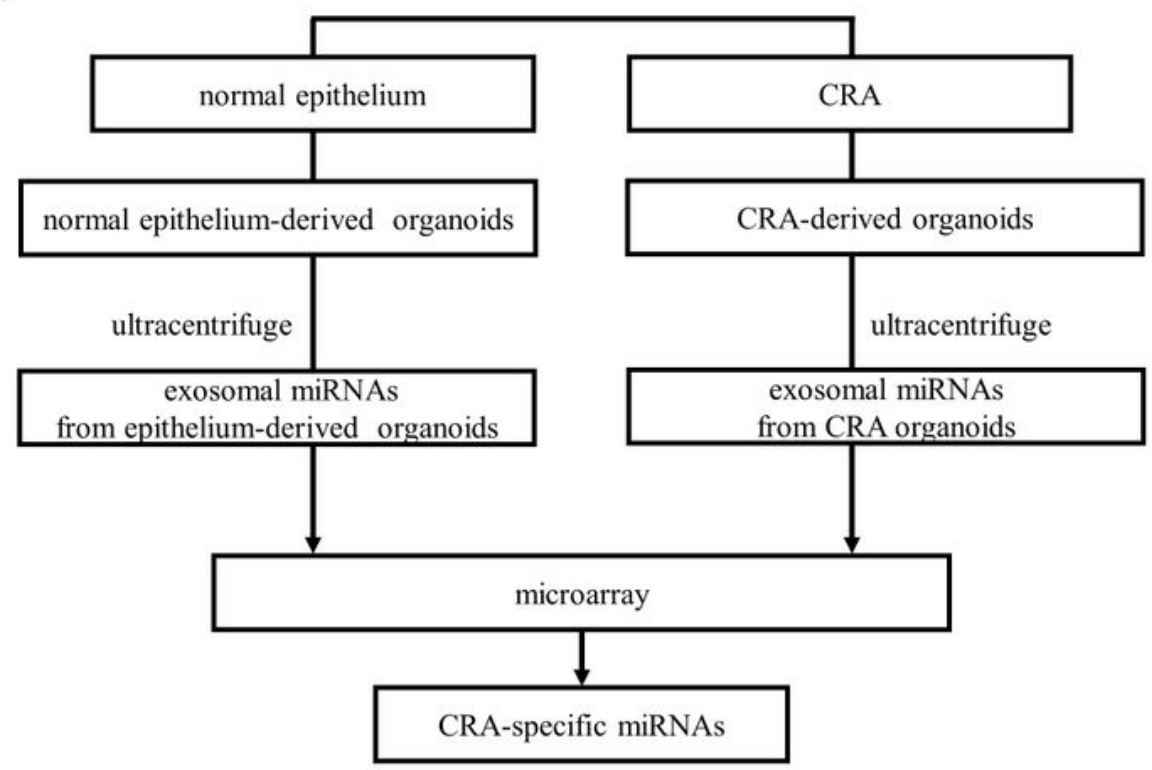

(B)
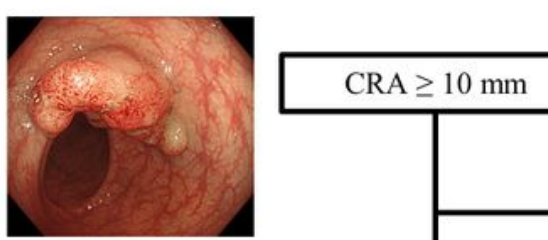

exclusion criteria

- past history of cancer

- inflammatory disease

- Vienna classification Category 5

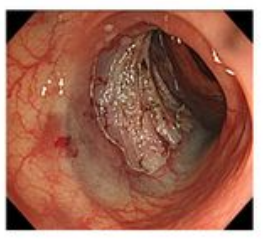

endoscopic treatment (ESD)

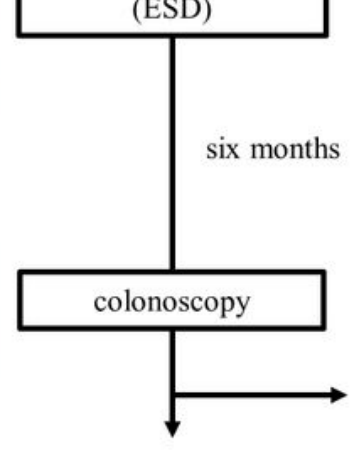

serum/exosomal miRNA by qRT-PCR

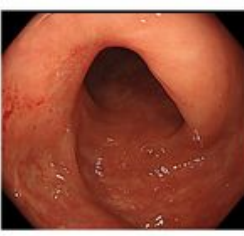

\section{colonoscopy}

Pre-treatment serum

$\underline{\text { Post-treatment serum }}$

\section{Figure 1}

Study design overview. (A) Selection of candidate miRNAs using organoid culture. (B) Flow diagram showing comparison of serum miRNAs before and after endoscopic resection by qRT-PCR: CRA, colorectal adenoma; miRNAs, microRNAs; ESD, endoscopic submucosal dissection; qRT-PCR, quantitative reverse transcription - polymerase chain reaction. 


\section{Figure 2}

\section{(A)}
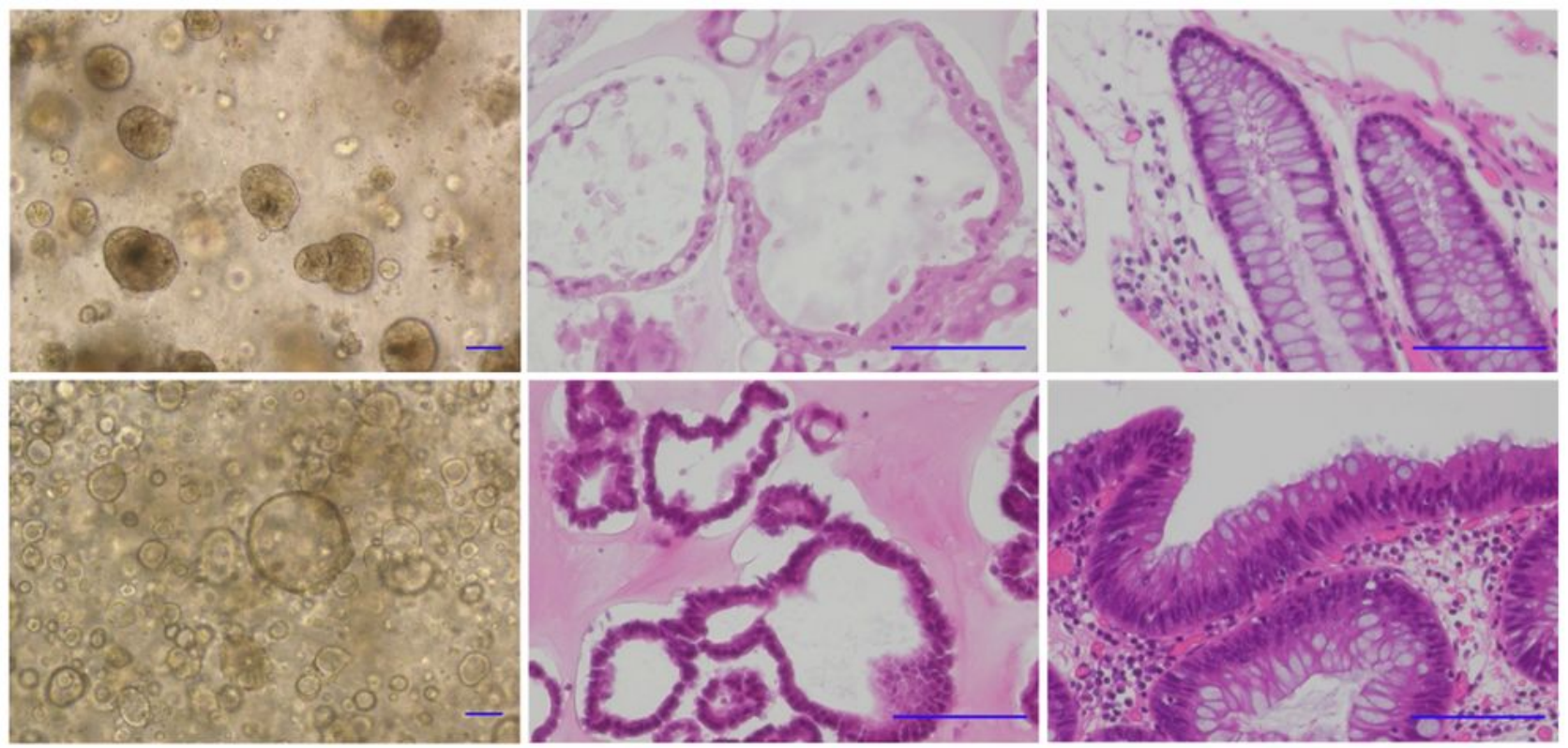

(B)
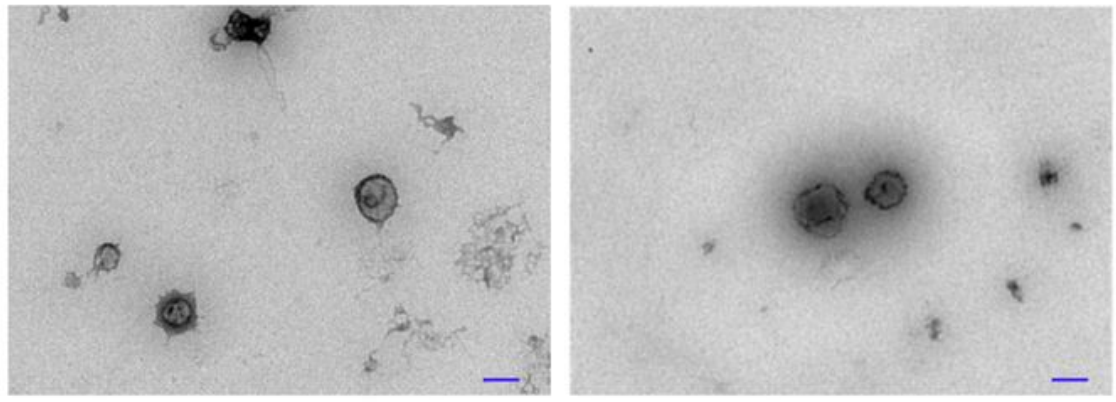

\section{(C)}
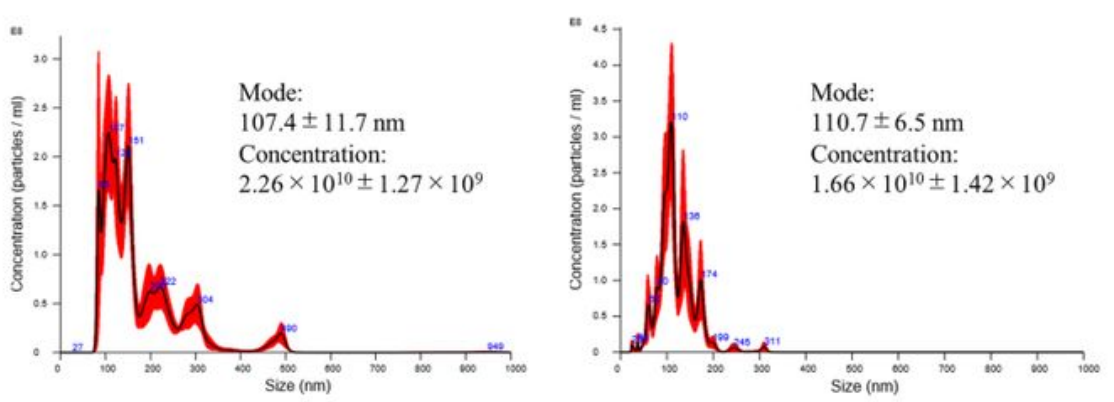

\section{Figure 2}

Patient-derived organoids and isolation of exosomes. (A) Top, images of normal colon epithelium. Optical microscopic image of organoids (left side), $\mathrm{H}$-E staining of organoids (center), and $\mathrm{H}$-E staining of endoscopically resected specimens (right side). Bottom, images of the CRA. Optical microscopic image of organoids (left side), H-E staining of organoids (center), and H-E staining of endoscopically resected specimens (right side). Each scale bar represents $100 \mu \mathrm{m}$. (B) Electron microscopic imaging of the 
isolated exosomes. Images of exosomes from normal colon epithelium-derived organoids (left) and of exosomes from CRA-derived organoids (right). Original magnification: $\times 15000$. Scale bar shows $100 \mathrm{~nm}$. (C) Size distribution of isolated exosomes analyzed using a Nanosight NS300. Size distribution of normal colon epithelium-derived organoids (left side). Size distribution of exosome from CRA-derived organoids (right side).

\section{Figure 3}

\section{(A)}

Exosomal miRNAs from normal colon epitheliumderived organoids

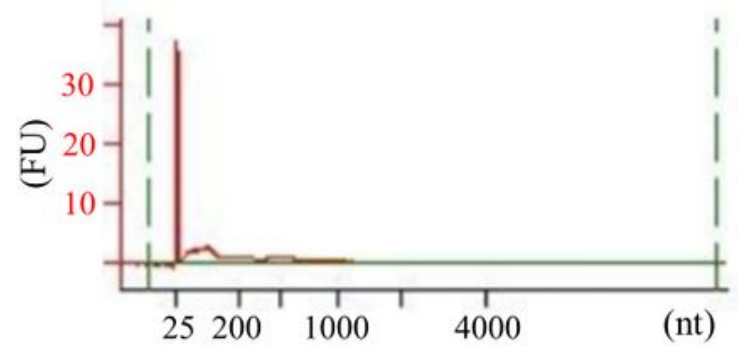

Exosomal miRNAs from CRA-derived organoids

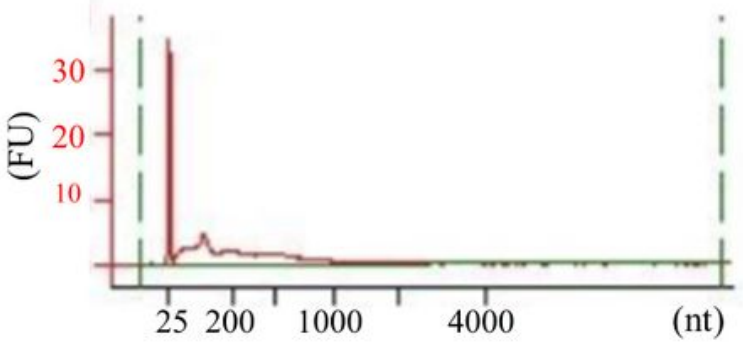

(B)

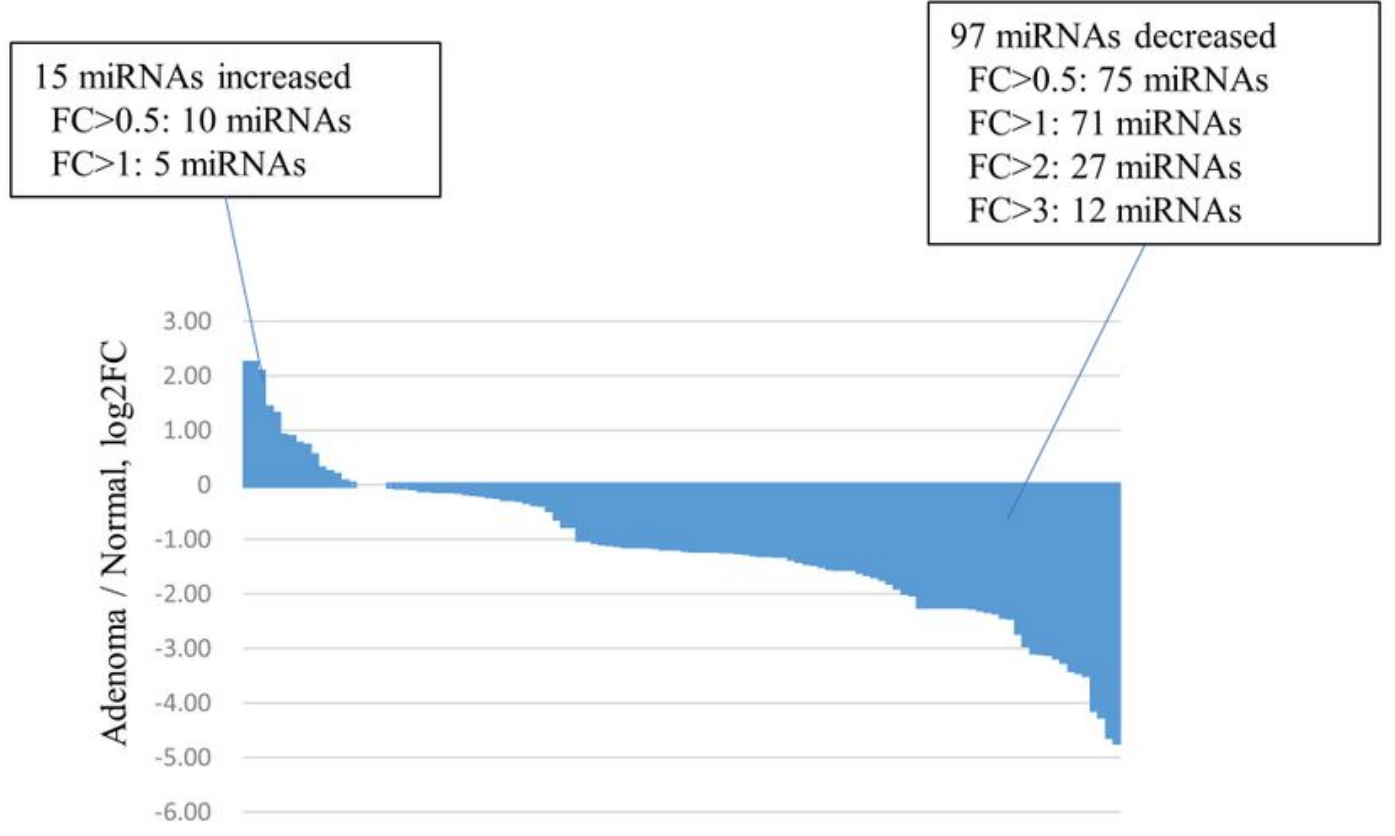

Figure 3 
Comprehensive analysis of exosomal miRNAs by microarray analysis. (A) Exosomal miRNAs were prepared from normal epithelium and CRA-derived organoids. Their integrity and quality were examined using a bioanalyzer system. Exosomal miRNA did not include ribosomal RNAs. Image of exosomal miRNAs from normal colon epithelium-derived organoids (left side), image of exosomal miRNAs from CRA-derived organoids (right side). (B) Agilent miRNAs microarray was used to compare miRNA expression profiles. MiRNAs with $|\log 2 \mathrm{FC}|>0.5$ were selected as differentially expressed. Exosomal miRNAs were prepared from normal epithelium and CRA-derived organoids. 10 miRNAs were found to be increased, whereas 75 miRNAs were found to be decreased in CRA: FC, fold change. 
Figure 4

(A)

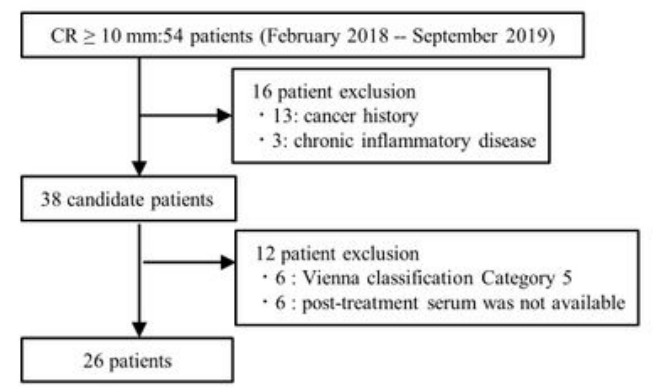

(B) Exosomal miRNAs
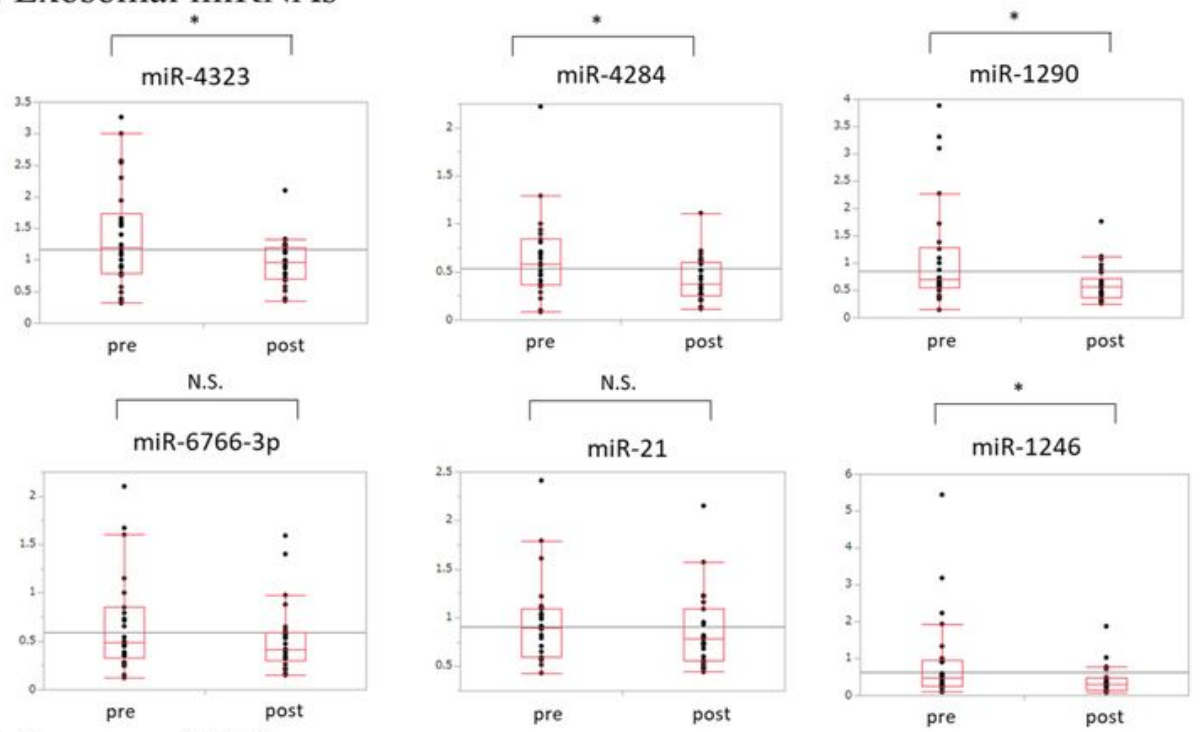

(C) Serum miRNAs
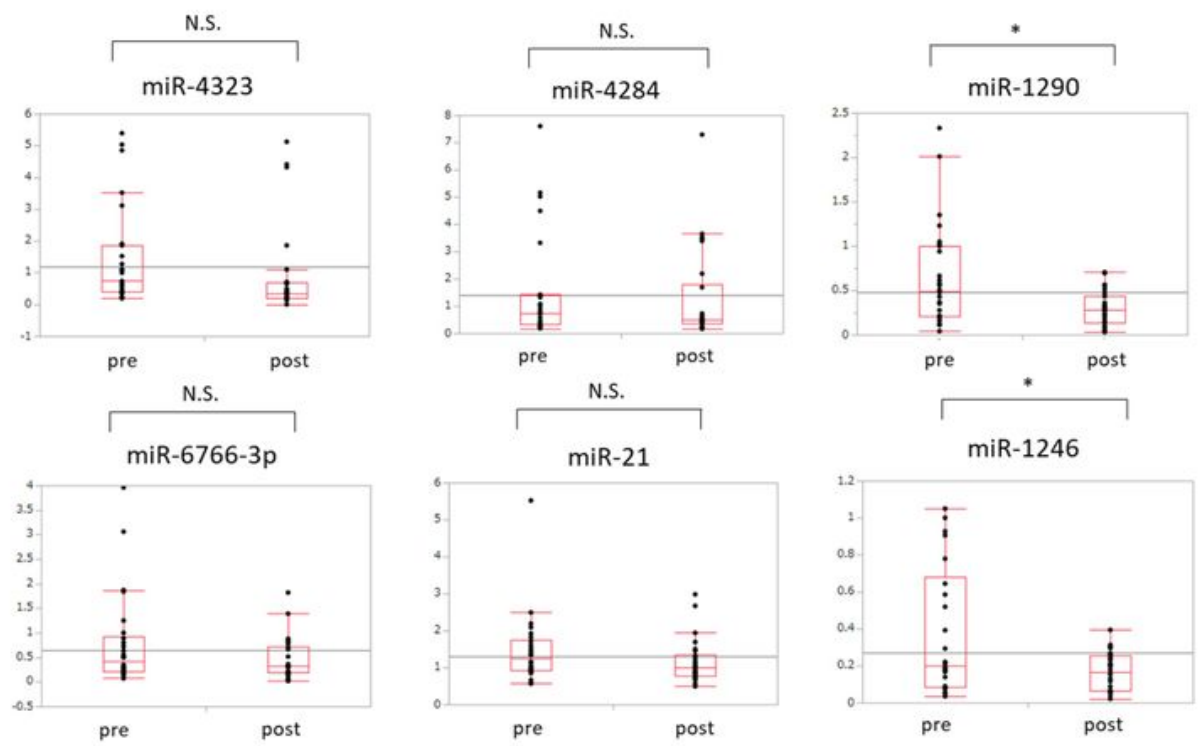

\section{Figure 4}

The expression levels of serum and exosomal miRNA before and after endoscopic resection. (A) Flow chart of the prospective study shows differences in miRNA expression before and after endoscopic resection. (B) Exosomal miRNAs expression levels before and after endoscopic resection in 26 patients. The respective expression levels of miR-4323, miR-4284, miR-1290, and miR-1246 were significantly lower after endoscopic resection. (C) Serum miRNA expression levels before and after endoscopic resection in 
26 patients. The expression levels of miR-1290 and miR-1246 were significantly lower after endoscopic resection: CRA, colorectal adenoma; Pre, before endoscopic resection; Post, after endoscopic resection; N.S., not significant; ${ }^{*}, p<0.05$.

\section{Figure 5}

(A) Exosomal miRNA

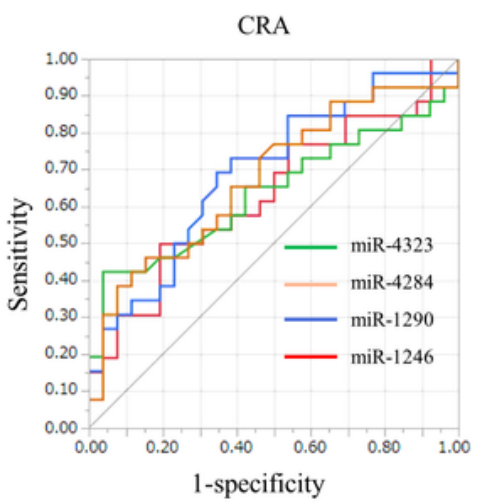

(C)

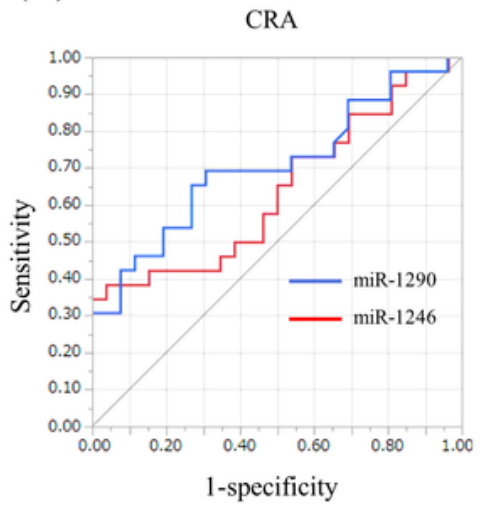

(B) Combination of 4 exosomal miRNAs

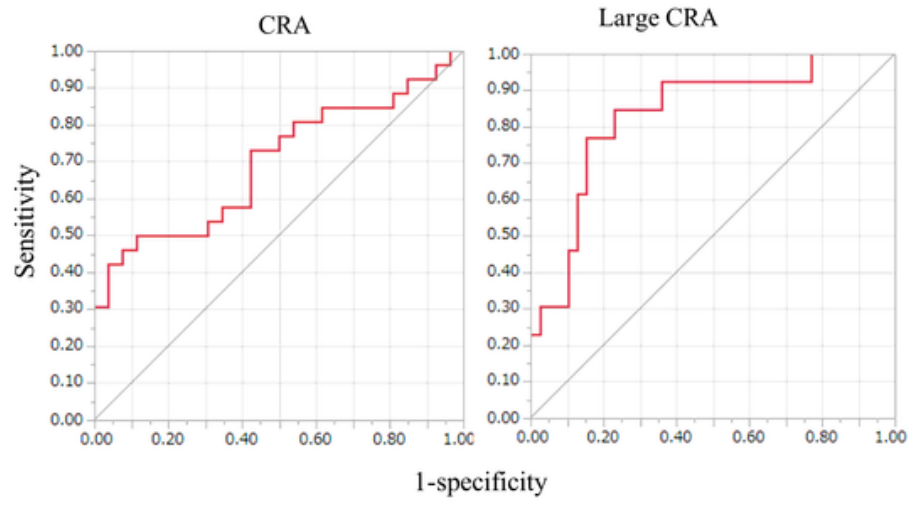

(D) Combination of 2 Serum miRNAs

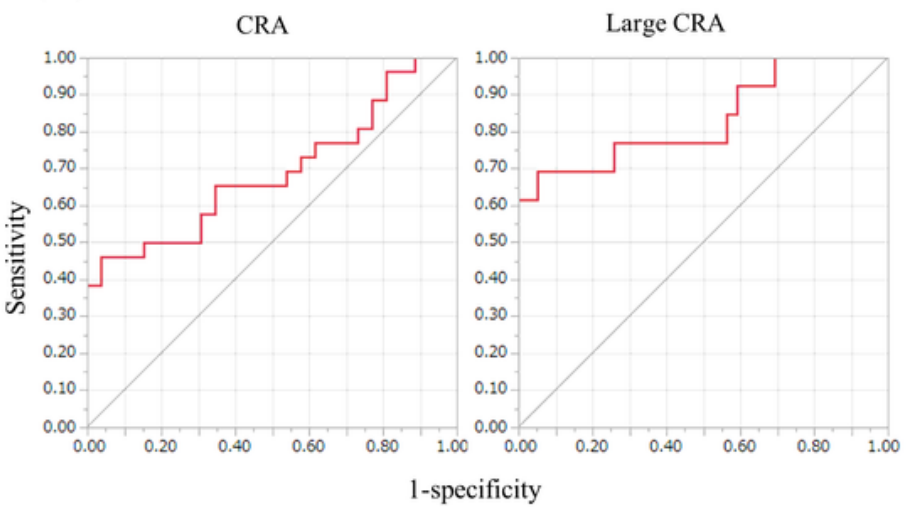

\section{Figure 5}

ROC curve analysis using serum or exosomal miRNAs levels to distinguish CRA patients. (A) Exosomal miR-4323, miR-4284, miR-1290, and miR-1246 levels yielded respective AUC values of 0.637, 0.677, 0.694, 
and 0.667 in distinguishing CRA. (B) Combined usage of four serum exosomal miRNAs gave AUC values of 0.698 in distinguishing CRA (left side), and 0.834 in distinguishing large CRA (approx. $\geq 12.6 \mathrm{~cm} 2$ ) (right side). (C) Serum miR-1290 and miR-1246 level yielded respective AUC values of 0.705 and 0.639 . (D) Combined usage of two serum miRNAs gave an AUC value for CRA as 0.691 (left side), and 0.834 for large CRA (right side): ROC, receiver operating characteristic; AUC, area under the curve; CRA, colorectal adenoma; green line, miR-4323; yellow line, miR-4284; blue line, miR-1290; red line, miR-1246. 